\title{
Oportunidades perdidas de imunização antitetânica de gestantes de Juiz de Fora, Minas Gerais, Brasil
}

\author{
Laura Maria Braga Borges de Mattos, ${ }^{1}$ Waleska Teixeira Caiaffa, ${ }^{2}$ \\ Ronaldo Rocha Bastos ${ }^{3}$ e Edward Tonelli ${ }^{4}$
}

RESUMO Objetivos. Estimar as oportunidades perdidas de vacinação antitetânica e a cobertura vacinal ao final da gravidez em gestantes de Juiz de Fora, Estado de Minas Gerais, Brasil.

Métodos. Entre $1^{\circ}$ de janeiro e 31 de agosto de 1996, realizou-se um estudo transversal com 430 mulheres selecionadas aleatoriamente entre todas as gestantes de Juiz de Fora. As participantes foram entrevistadas a respeito do controle pré-natal, conhecimento sobre o tétano e vacinação antitetânica.

Resultados. A idade das gestantes variou de 14 a 45 anos (média de 26,4 \pm 6,9 anos); 420 eram de zona urbana e 10 de zona rural. O controle pré-natal foi feito pelo Sistema Único de Saúde em 69,5\% (299) das mulheres; 27,6\% (119) utilizaram outros convênios; e 2,8\% (12) não fizeram controle pré-natal. Trezentas e cinqüenta e duas mulheres $(81,8 \%)$ portavam o cartão de controle pré-natal e $85,6 \%$ (368) tiveram quatro ou mais consultas pré-natais. Com relação ao conhecimento do tétano, 92,1\% (396) demonstraram conhecer a doença e sua gravidade. Dentre 430 gestantes, 359 (83,5\%) iniciaram a gestação não imunizadas contra o tétano: 104 foram imunizadas durante a gravidez em estudo e 255 permaneceram sem vacina até o puerpério, apesar do aumento significativo na chance de imunização com o aumento do número de visitas de pré-natal $(\mathrm{OR}=2,7$ para quatro ou mais visitas; $\mathrm{P}<0,001)$. Foram de $70 \%$ as oportunidades perdidas de imunização antitetânica na gestação e de 40,6\% a cobertura vacinal das gestantes. Conclusão. A baixa cobertura vacinal, inferior aos níveis recomendados pela Organização Mundial da Saúde, e o índice elevado de oportunidades perdidas de imunização antitetânica apontam para a necessidade de se instituir uma estratégia de incentivo à vacinação, direcionada aos profissionais de saúde em geral e especialmente àqueles responsáveis pelo atendimento das gestantes, tanto em postos de saúde quanto em clínicas privadas. Além disso, a rotina de vacinação em Juiz de Fora deve ser intensificada.

Palavras-chave Tétano, complicações infecciosas na gravidez, vacinação.

Universidade Federal de Juiz de Fora, Faculdade de Medicina, Departamento Materno-Infantil, Juiz de Fora (MG), Brasil. Esta autora faleceu em 18 de abril de 1998.

2 Universidade Federal de Minas Gerais, Faculdade de Medicina, Departamento de Medicina Preventiva e Social, Belo Horizonte (MG), Brasil. Correspondência e pedidos de separatas devem ser encaminhados a esta autora no seguinte endereço: Faculdade de Medicina da UFMG, Avenida Alfredo Balena 190, $10^{\circ}$ andar, CEP 30130-100, Belo Horizonte, MG, Brasil. E-mail: wcaiaffa@medicina. ufmg.br

3 Universidade Federal de Juiz de Fora, Instituto de Ciências Exatas, Departamento de Estatística.
O tétano é uma doença infecciosa, não contagiosa, causada pela ação de uma exotoxina produzida pelo Clostridium tetanii sobre o sistema nervoso central (CNS) (1). É uma doença de fácil prevenção que, anualmente, causa cerca de 309000 mortes. São motivo de

\footnotetext{
4 Universidade Federal de Minas Gerais, Faculdade de Medicina, Departamento de Pediatria.
}

preocupação especial o tétano neonatal e o tétano materno, ambos evitáveis através da imunização da mãe durante a gestação. Estima-se que, no ano de 2000, o tétano neonatal sozinho foi responsável por 200000 mortes em 57 países, estando $90 \%$ dessas mortes concentradas em 27 países (2). Em algumas comunidades pouco desenvolvidas, o tétano ainda é responsável pela maioria das mortes neonatais. Na Etió- 
pia, por exemplo, é responsável por $63 \%$ dessas mortes (3). No Brasil, estima-se em 400 o número de casos de tétano neonatal por ano (4). Existe uma iniciativa mundial para a eliminação (definida como a ocorrência de menos de um caso de tétano neonatal por 1000 nascidos vivos) do tétano materno e neonatal até o ano de 2005 (2).

O bacilo causador do tétano é largamente encontrado sob a forma de esporo, sobretudo no solo (terra, areia, poeira de rua), nas fezes de animais herbívoros, em águas putrefeitas, em pregos e latas enferrujados e em plantas. Além disso, entre 20 e $30 \%$ dos seres humanos são portadores intestinais assintomáticos de esporos tetânicos. Devido à ubiqüidade do bacilo, as fontes de contágio são inúmeras, tornando difícil o seu controle e deixando o ser humano naturalmente exposto ao contágio (1).

Várias medidas profiláticas têm sido propostas para o controle e erradicação do tétano. No entanto, a eficácia e a efetividade dessas medidas ainda estão em debate. Resta, portanto, a imunização ativa, que, seguramente, é a medida mais eficaz e prática recomendada (5).

A vacina antitetânica foi produzida pela primeira vez por Ramon, em 1923. É considerada uma das vacinas mais efetivas e seguras dentre as de utilização corrente (6). No Brasil, o Programa Nacional de Imunizações (PNI), instituído em 1973, regulamenta a uniformidade do uso da vacina antitetânica e de outras vacinas em todo o território nacional e tem como um de seus objetivos a erradicação do tétano (7). Esse programa estabelece como prioritária a aplicação do toxóide tetânico (TT) ou da vacina dupla tipo adulto (dT) em gestantes. A orientação é de se aplicarem três doses (TT ou dT) até o $8^{\circ}$ mês em gestantes não imunizadas, com intervalo de 30 a 60 dias entre cada dose. Em gestantes incompletamente imunizadas, orienta-se completar as três doses antes do $8^{\circ}$ mês. Em gestantes previamente imunizadas, se a última dose foi aplicada há mais de 5 anos, deve-se administrar uma dose de reforço até o $8^{\circ}$ mês. Em geral, todos os adultos devem receber uma dose de reforço da vacina antitetânica a cada 10 anos (8).
O controle de uma doença imunoprevenível é geralmente alcançado com cobertura vacinal de 70 a $80 \%$ em uma população suscetível. Uma das estratégias para ampliar a cobertura vacinal é o aproveitamento das oportunidades de se vacinar crianças e adultos durante as visitas desses indivíduos a um serviço de atenção à saúde (9). Marques (5) afirma que, para se conseguir uma boa cobertura vacinal, todo adulto ou criança sem contra-indicações não deve sair de uma instituição de saúde sem ser vacinado. Nesse contexto, a não vacinação em situações favoráveis - conhecida como oportunidade perdida de vacinação - pode ser definida amplamente e de forma operacional como a taxa de suscetíveis necessitando a vacinação em relação à população alvo (10).

A erradicação do tétano neonatal, através da garantia de vacinação antitetânica para todas as gestantes, é uma das metas de saúde da criança definidas pela OMS e pelo Fundo das Nações Unidas para a Infância (UNICEF) (11), e a perda de oportunidades de aplicar essa vacina durante a gestação pode ser caracterizada como uma oportunidade perdida de vacinação. Em abril de 2002, UNICEF anunciou que 15000 recém-nascidos foram salvos em 2001 devido ao reforço da campanha para a eliminação do tétano neonatal no mundo, consoante com o objetivo da instituição de erradicar o tétano neonatal e materno até $2005(2,12)$.

A partir dessa perspectiva, o presente estudo objetivou estimar as oportunidades perdidas de vacinação em gestantes e levantar a cobertura vacinal das gestantes por ocasião do parto em Juiz de Fora, uma cidade de porte médio no Estado de Minas Gerais, Brasil.

\section{MATERIAIS E MÉTODOS}

Realizou-se um estudo do tipo transversal ou de prevalência em amostra aleatória de gestantes no ano de 1996. Assumindo-se valores de $50 \%$ para a proporção prevista de cobertura vacinal, nível de significância de 5\%, precisão absoluta de $10 \%$, efeito relacionado ao desenho da amostra de até 2 e perda de
$10 \%$, adotou-se como tamanho mínimo de amostra 431 observações (13).

Para a coleta do material foi utilizado o método de janelas de tempo, que permite economia de tempo e pessoal para a pesquisa e evita o viés de sazonalidade e de tempo (14). Com esse método, todas as gestantes de parto hospitalar (99,8\% do total de gestantes da cidade) tiveram chances de participar da amostra. O período de tempo projetado para o estudo foi de $1^{\circ}$ de fevereiro a 31 de agosto de 1996, totalizando 30 semanas, das quais 13 semanas foram escolhidas aleatoriamente (janelas de tempo). Em cada uma dessas semanas, 33 gestantes foram aleatoriamente selecionadas para a amostra. Diariamente, o pesquisador responsável comparecia a todos os hospitais da cidade que admitiam gestantes para trabalho de parto. Nesses hospitais eram verificadas as listas da portaria, das enfermarias e registros de apartamentos. A gestante sorteada era convidada a participar e recebia esclarecimentos sobre a pesquisa. A entrevista era realizada conforme questionário padrão, após consentimento livre e esclarecido. Ao final da entrevista, todas as gestantes recebiam orientação sobre a vacinação antitetânica e outros cuidados relacionados ao período pré-natal e puerpério.

As variáveis estudadas foram: vacinação antitetânica durante a gestação, escolaridade, relato do conhecimento da doença, tipo de assistência durante o pré-natal e número de consultas no cartão de pré-natal. As informações foram verificadas no cartão de prénatal em $81,8 \%$ (352) das gestantes. No caso de 78 entrevistadas, tais informações foram obtidas apenas através da resposta ao questionário, já que essas mulheres não possuíam o cartão de pré-natal ou não estavam com ele no momento da entrevista.

Os dados foram processados utilizando-se o programa Epi Info 6.04 (15). A associação entre a imunização antitetânica e as variáveis estudadas foi considerada significativa para $P \leq$ 0,05 , conforme o teste do qui-quadrado $\left(\chi^{2}\right)$ e o teste exato de Fisher, quando necessário. A razão de chances (odds ratio, OR) foi calculada com os respectivos intervalos de confiança de 95\% (IC95\%). 
O cálculo de oportunidades perdidas de vacinação (OPV) antitetânica em gestantes foi feito pela fórmula $\mathrm{OPV}=$ número de gestantes não vacinadas/número de gestantes que deveriam ter sido vacinadas (16). O cálculo da cobertura vacinal (CV) foi feito utilizando-se a fórmula adaptada da Secretaria de Saúde do Estado de Minas Gerais (17): CV = gestantes que receberam pelo menos uma dose de vacina antitetânica há menos de 5 anos/ número de gestantes pesquisadas.

\section{RESULTADOS}

A amostra final de 430 gestantes correspondeu a 2,2 vezes o valor exigido para uma amostra aleatória simples $(\mathrm{n}=196)$. Os testes de aderência $\left(\chi^{2} \mathrm{e}\right.$ teste de proporções) mostraram não haver diferenças estatisticamente significativas entre a população estudada e a população de onde a amostra foi retirada em termos das características selecionadas (dados não mostrados).

Das 430 gestantes, $395(91,9 \%)$ eram residentes em Juiz de Fora e 35 (8,1\%) eram procedentes de municípios vizinhos; $420(97,7 \%)$ eram residentes em zona urbana e $10(2,3 \%)$ em zona rural. A idade das gestantes variou de 14 a 45 anos, com média de 26,4 $\pm 6,9$ anos. Encontra-se na tabela 1 a distribuição das gestantes de acordo com escolaridade, tipo de atendimento durante o controle pré-natal, conhecimento sobre o tétano e status da vacinação antitetânica. A escolaridade média em anos de freqüência à escola foi de 6,6 6 3,7 anos, com mediana de 6 anos. Dois terços das participantes relataram 8 anos ou menos de freqüência à escola. Verificando o conhecimento sobre o tétano, observou-se que a grande maioria das gestantes (396) sabia que o tétano é letal e que poderia acometer o recém-nascido como o mal dos 7 dias.

Das 430 gestantes, 359 iniciaram a gestação em estudo não imunizadas para o tétano; 71 (16,5\%) já eram imunes por terem sido vacinadas adequadamente há menos de 5 anos (32 em gestação anterior e 39 por outros motivos). Cento e quatro $(24,2 \%)$ gestantes foram vacinadas durante a gestação e,
TABELA 1. Características das gestantes que participaram do estudo sobre oportunidades perdidas de vacinação antitetânica, Juiz de Fora (MG), Brasil, 1996

\begin{tabular}{lc}
\hline \multicolumn{1}{c}{ Característica } & Amostra (\%) \\
\hline Escolaridade & 2,0 \\
Nenhuma & 64,7 \\
$1^{\circ}$ grau incompleto (menos de 8 anos) & 15,3 \\
$1^{\circ}$ grau completo (8 anos completos) & 10,2 \\
$2^{\circ}$ grau completo (11 anos completos) & 7,6 \\
Superior (16 ou mais anos) & \\
Controle pré-natal & 69,5 \\
Sistema Único de Saúde (público) & 27,6 \\
Convênio ou privado & 2,8 \\
Não realizaram & \\
Conhecimento da doença tétano & 92,1 \\
Sim & 7,9 \\
Não & \\
Imunização antitetânica & 9,1 \\
Imunizadas fora da gestação & 7,4 \\
Imunizadas em gestação anterior & 24,2 \\
Imunizadas na gestação em estudo & 59,3 \\
Não imunizadas & \\
\hline a $n=430$. &
\end{tabular}

portanto, $255(59,3 \%)$ permaneceram não imunizadas até o final da gestação.

Com relação à freqüência das mulheres às consultas de pré-natal: 12 mulheres $(2,8 \%)$ não fizeram pré-natal, $23(5,3 \%)$ compareceram a uma ou duas consultas, $27(6,3 \%)$ compareceram a três consultas e $368(85,6 \%)$ compareceram a quatro consultas ou mais. A tabela 2 mostra a relação entre o número de consultas e a imunização antitetânica até o final da gestação. Observou-se uma associação estatisticamente significativa entre o número de consultas e a imunização antitetânica $\left(\chi^{2}=9,85 ; P=0,0016\right)$. Gestantes com quatro ou mais visitas ao prénatal tinham duas vezes mais chances de estarem imunizadas contra o tétano do que gestantes com zero a três visitas (OR = 2,67; IC95\% = 1,36 a 5,30). Vale lembrar, entretanto, que, das 255 gestantes não imunizadas, 207 compareceram a quatro ou mais consultas de pré-natal.

Não foi encontrada associação estatisticamente significativa entre estar imunizada e ter 6 ou mais anos de escolaridade $\left(\chi^{2}=1,39 ; P=0,238\right)$; ser atendida pelo SUS $\left(\chi^{2}=0,12 ; P=0,730\right)$ e conhecer a doença tétano $\left(\chi^{2}=3,10\right.$; $P=0,078$ ).

Assumindo que 243 gestantes (255 que não receberam vacina menos 12 gestantes que não compareceram ao pré-natal) não foram vacinadas contra o toxóide tetânico quando, de fato, tinham indicação da vacina, em um universo de 349 (359 menos 12) gestantes que compareceram a pelo menos uma consulta de pré-natal, estimaram-se em $70,0 \%$ as oportunidades perdidas de imunização durante a gestação, neste estudo.
TABELA 2. Associação entre o número de consultas no pré-natal e a imunização antitetânica até o final da gestação, Juiz de Fora (MG), Brasil, 1996a

\begin{tabular}{|c|c|c|c|c|c|c|}
\hline \multirow{3}{*}{$\frac{\text { Consultas no pré-natal }}{0 \text { a } 3}$} & \multicolumn{6}{|c|}{ Imunização antitetânica até o final da gestação } \\
\hline & \multicolumn{2}{|c|}{ Não (\%) } & \multicolumn{2}{|c|}{$\operatorname{Sim}(\%)$} & \multicolumn{2}{|c|}{ Total } \\
\hline & 48 & $(77,0)$ & 14 & $(23,0)$ & 62 & $(100,00)$ \\
\hline 4 ou mais & 207 & $(56,0)$ & 161 & $(44,0)$ & 368 & $(100,00)$ \\
\hline
\end{tabular}

a OR $=2,67 ; \mathrm{IC} 95 \%=1,36$ a 5,$30 ; \chi^{2}=9,85 ; P<0,001$. 
Considerando que 175 gestantes haviam recebido pelo menos uma dose de vacina antitetânica há menos de 5 anos, a cobertura vacinal em gestantes de Juiz de Fora foi estimada em 40,6\%.

\section{DISCUSSÃO}

Este estudo demonstra que $70 \mathrm{em}$ cada 100 gestantes que deveriam ter sido vacinadas contra tétano não o foram por ocasião do pré-natal. Além disso, somente $40,6 \%$ das gestantes observadas haviam recebido pelo menos uma dose de vacina antitetânica há menos de 5 anos em uma amostra representativa de todas as gestantes de Juiz de Fora em 1996.

Existem duas importantes diferenças entre o tétano e as outras doenças cuja prevenção está incluída no PNI. O primeiro ponto é que, para o tétano, a imunização só é alcançada mediante vacinação, já que a imunidade não pode ser naturalmente adquirida. O segundo aspecto é que, nesse caso, temos duas faixas da população para imunização: as crianças e as mulheres em idade fértil (17).

Estudos mais antigos demonstraram variações entre 41 e $76 \%$ em termos de oportunidades perdidas de vacinação contra o tétano: $41 \%$ na Etiópia, $44 \%$ em Camarões, 49\% na Turquia, 69\% no Paquistão, 57\% na Índia, 54\% no Nepal e $76 \%$ na Indonésia (16). Mais recentemente, a Etiópia registrou uma freqüência menor de oportunidades perdidas de vacinação, variando de 4 a 13\% dependendo da procedência, urbana ou rural, da gestante (4). Em contraste, na Nigéria, mesmo recentemente, o índice de oportunidades perdidas de vacinação ainda é alto, de $66 \%$ (18).

Nas Américas, o quadro não é muito diferente. Estudos feitos através de entrevista com acompanhantes de crianças e mulheres em idade fértil na saída da unidade de saúde mostram percentuais que variam de 50 a $80 \%$ de oportunidades perdidas de vacinação em gestantes e mulheres em idade fértil: 50\% em El Salvador, 52\% no Equador, $80 \%$ na Guatemala e $72 \%$ na Venezuela (16).
As causas apontadas para as oportunidades perdidas têm sido aquelas ligadas ao serviço de saúde e aos fatores individuais dos grupos populacionais que os freqüentam. Destacam-se no primeiro grupo as contra-indicações orientadas pelo pessoal de saúde; falta de recursos; deficiências na organização dos serviços; atitude do pessoal de saúde frente à vacinação e resposta da população à vacinação $(9,16,18-22)$.

No que se refere a determinantes individuais, o baixo nível de escolaridade constitui um nítido fator de exclusão social. A pouca escolaridade materna vem sendo apontada como um fator associado à menor completude de vacinação em estudos sobre os determinantes das falhas de vacinação em crianças (19-21).

Contrariamente, neste estudo, a melhor escolaridade, o relato de receber o atendimento do pré-natal fora do sistema público e o conhecimento acerca da doença não foram variáveis associadas à maior imunização antitetânica. Um possível fator de confusão poderia ser o fato de que $98 \%$ da população entrevistada residia em zona urbana, com acesso aos serviços de saúde, fator também descrito como associado à maior completude vacinal tanto em gestantes como crianças $(4,21)$.

O grande diferencial, neste estudo, foi representado pelo número de consultas de pré-natal, sugerindo que, à medida que a gestante comparecia às consultas, cresciam suas chances de ser imunizada. Portanto, um dos fatores decisivos para elevar a cobertura vacinal antitetânica na gestante poderia estar relacionado à participação de profissionais de saúde que prestam assistência nos serviços de pré-natal. Estes resultados são compatíveis com as conclusões de outro estudo que mostraram que quanto mais cedo se iniciava a visita de crianças aos serviços de saúde, maiores eram as oportunidades de prevenção (21). Sob esse aspecto, torna-se adequada outra abordagem, cada vez mais usada nos estudos de oportunidades perdidas de prevenção: a avaliação da efetividade dos serviços de saúde a partir da investigação acerca da contribuição dos profissionais para a prevenção e pro- moção da saúde, não só em doenças imunopreveníveis, mas também em outras intervenções preventivas (22).

Apesar do nível baixo de cobertura vacinal encontrado neste estudo, ele foi maior do que os índices registrados pelo Serviço Municipal de Vigilância Epidemiológica de Juiz de Fora (em 13 de março de 1998) em gestantes, que variou de 6,6\% em 1994 a 13,6\% em 1997. Resta lembrar que as informações provenientes desse serviço foram colhidas em relatórios dos postos de saúde e, portanto, são relativas somente às gestantes atendidas pelo SUS, não abrangendo cerca de $30 \%$ das gestantes da cidade, que são atendidas pelos serviços suplementares. Além disso, é importante ressaltar que houve ainda um percentual importante de mulheres (48\%) que, mesmo comparecendo ao serviço de assistência prénatal quatro ou mais vezes, deixou de receber a vacina antitetânica.

Este estudo apresenta limitações devido a seu desenho transversal, já que, apesar de podermos identificar associações entre as variáveis, não podemos determinar a temporalidade dos acontecimentos. Por exemplo, embora possamos constatar a associação estatisticamente significativa entre número de consultas no pré-natal e vacinação antitetânica, não podemos afirmar se é a maior freqüência ao prénatal que determina a vacinação ou se as gestantes que recebem a vacina são mais cuidadosas e por esse mesmo motivo comparecem mais vezes ao serviço de pré-natal.

Apesar dessas limitações, em Juiz de Fora, a perda de $70 \%$ das oportunidades de vacinação e a baixa cobertura vacinal antitetânica em gestantes apontam para a necessidade de conscientização de todo o pessoal de saúde, em especial do pré-natalista e do serviço de enfermagem, principais responsáveis pelo atendimento das gestantes, tanto nos postos públicos quanto em clínicas privadas. Além disso, seriam recomendáveis estudos que esclarecessem o porquê dessas oportunidades perdidas de imunização de tétano na região, contribuindo assim, simultaneamente, para a erradicação do tétano neonatal e do tétano acidental em mulheres. 


\section{REFERÊNCIAS}

1. Focaccia R. Tétano. Em: Farhat CK, Carvalho ES, Carvalho LHFR, Succi RCM. Infectologia pediátrica. $2^{a}$ ed. São Paulo: Atheneu; 1999. Pp. 335-340.

2. Centers for Disease Control and Prevention. Progress toward the global elimination of neonatal tetanus, 1989-1993. MMWR 1994;43: 885-887, 893-894.

3. Mekonnen Y. Missed opportunity of tetanus toxoid immunization among pregnant women in Southern Ethiopia. Ethiop J Health Dev 2000;14(2):143-148.

4. Barros FC, Victora CG. Epidemiologia da saúde infantil. $2^{\text {a }}$ ed. São Paulo: HucitecUNICEF; 1994.

5. Marques AN. Pediatria social - teoria e prática. $1^{a}$ ed. Rio de Janeiro: Cultura Médica; 1986.

6. Focaccia R, Veronesi R. Tétano. Em: Farhat CK. Fundamentos e prática das imunizações em clínica médica e pediatria. $3^{\mathrm{a}}$ ed. Rio de Janeiro: Livraria Atheneu Ltda; 1989. Pp. 65- 73.

7. Fischmann A, Santos AR. Vigilância epidemiológica e controle de doenças transmissíveis. Em: Tonelli E, Freire LMS. Doenças infecciosas na infância e adolescência. $2^{\mathrm{a}}$ ed. Rio de Janeiro: MEDSI; 2000. Pp. 175-212.

8. Brasil, Ministério da Saúde, Fundação Nacional da Saúde. Manual de procedimentos para vacinação. Brasília: Ministério da Saúde; 1994.
9. Loevinsohn BP. Missed opportunities for immunization during visits for curative care practical reasons for their occurrence. Am J Trop Med Hyg 1989;41(3):255-258.

10. Rey M. Resurgence of diphtheria in Europe. Clin Microbiol Infect 1996;2(1):71-72.

11. Sociedade Brasileira de Pediatria, Comitê de Cuidados Primários. Manual de cuidados primários. São Paulo: SBP; 1994

12. UNICEF. Campanha contra o tétano materno e neonatal. Disponível em: http://www. unicef.pt/noticias/noticias14.html. Acessado em 12 julho 2003.

13. Lwanga SK, Lemeshow S. Determinacion del tamaño de las muestras en los estudios sanitários. Ginebra: Organización Mundial de la Salud; 1991.

14. Jeannel D, Niel G, Costagliola D, Danis M, Traore BM, Gentilini M. Epidemiology of toxoplasmosis among pregnant women in Paris area. Int J Epidemiol 1988;17(3):595-602.

15. Dean AG, Dean JA, Coulombier D, Brendel $\mathrm{KA}$, Smith DC, Burton AH, et al. Epi Info, Version 6.0: a Word-processing, database and statistics program for public health on IBMcompatible microcomputers. Atlanta: Centers for Disease Control and Prevention; 1995.

16. Olivé JM, De Quadros C, Castillo C. Oportunidades perdidas de vacunación en las Americas: diagnóstico e intervenciones, 1988-1990. Documento apresentado na IX Reunión del
GTA sobre Enfermedades Prevenibles por Vacunación; 12-15 março 1991;9:91-95.

17. Minas Gerais, Secretaria de Saúde do Estado de Minas Gerais. Plano de ação quinquenal para eliminação do tétano neonatal no estado de Minas Gerais. Belo Horizonte: Secretaria da Saúde; 1991.

18. Edet EE, Ikpeme BM, Ndifon WO, Oyo-Ita AE. Factors associated with missed opportunities to immunize with tetanus toxoid at a tertiary health institution in Nigeria. Centr Afr J Med 1998;44(8):199-202.

19. Cutts FT, Diallo S, Zell ER, Rhodes P. Determinants of vaccination in an urban population in Conakry, Guinea. Int J Epidemiol 1991;20(4):1099-1106.

20. Ross A, Kennedy AB, Holt E, Guyer B, Hou $W$, Hughart N. Initiating the first DTP vaccination age-appropriately: a model for understanding coverage. Pediatrics 1998;101(6):970974

21. Deshpande R, Nimbalkar S, Banker N, Kapoor A. Prevalence of missed opportunities for measles immunization in rural areas of Gujarat. Indian J Pediatr 2001;68(7):609-612.

22. Brull R, Ghali WA, Quan H. Missed opportunities for prevention in general internal medicine. CMAJ 1999;160(8):1137-1140.

Manuscrito recebido em 14 de novembro de 2002. Aceito em versão revisada em 30 de julho de 2003.

Objective. To estimate the missed opportunities for tetanus vaccination and to determine the immunization coverage at the end of pregnancy in women living in the city of Juiz de Fora, which is in the state of Minas Gerais, Brazil.

Missed opportunities for tetanus immunization of pregnant women in Juiz de Fora, Minas Gerais, Brazil
Methods. A cross-sectional study was carried out of 430 women who were randomly selected from all the maternity hospitals in the city, from February through August 1996. The women were interviewed regarding prenatal care, their knowledge of tetanus, and tetanus immunization before or during the latest pregnancy.

Results. The age of the participants ranged from 14 to 45 years (mean $=26.4 \pm 6.9$ years); 420 women lived in urban areas and 10 in rural areas. Prenatal care was from the Brazilian public Unified Health System in $69.5 \%$ of the women (299 of 430$) ; 27.6 \%$ (119) received prenatal care through other types of providers; and $2.8 \%$ (12) did not receive prenatal care. Of the 430 participants, 352 of them $(81.8 \%)$ had their prenatal care card with them during the interview; $85.6 \%$ (368) had four or more prenatal care visits. Regarding their knowledge of tetanus, $92.1 \%$ (396) knew about the disease and its severity. Out of the 430 women, 359 of them $(83.5 \%)$ started the latest pregnancy without having had a tetanus vaccination; 104 were vaccinated during the latest pregnancy, so 255 had not been vaccinated by the time of delivery. This was in spite of the significant increase in the opportunities for being vaccinated with an increase in the number of prenatal visits. The odds ratio for pregnant women with four or more prenatal visits being vaccinated by the end of the pregnancy was 2.7 times that of the women with zero to three visits. Seventy percent of the opportunities for tetanus immunization were missed in the study population. The overall tetanus immunization coverage was $40.6 \%$ by the end of pregnancy.

Conclusions. The low immunization coverage was below the level recommended by the World Health Organization. In combination with the high rate of missed opportunities for vaccination, this points to the need to implement a tetanus vaccination promotion strategy directed at health professionals in general but especially at those who provide care for pregnant women, in both public and private health clinics. In addition, the vaccination practices in Juiz de Fora should be strengthened. 